\title{
Erratum to: Determinants of self-employment survival in Europe
}

\author{
José María Millán · Emilio Congregado • \\ Concepción Román
}

Published online: 11 June 2010

(C) Springer Science+Business Media, LLC. 2010

\section{Erratum to: Small Bus Econ \\ DOI 10.1007/s11187-010-9260-0}

Results and descriptive statistics concerning the countries Greece and Germany have been mistakenly interchanged. This applies to results in Table 1 (subsection 5.1), descriptive statistics in Table 4 (Appendix), and the last paragraphs on subsections 5.1 and 5.2, where the references to Greece correspond to Germany.

In addition, the definitions of the dependent variables concerning single and competing risk models in the Appendix have also been mistakenly interchanged.

The authors sincerely apologize to all readers for any inconveniences caused by these mistakes.
The online version of the original article can be found under doi:10.1007/s11187-010-9260-0.

Electronic supplementary material The online version of this article (doi:10.1007/s11187-010-9289-0) contains supplementary material, which is available to authorized users.

J. M. Millán $(\bowtie) \cdot$ E. Congregado · C. Román

Universidad de Huelva, Huelva, Spain

e-mail: jose.millan@dege.uhu.es
5 Results

$[\ldots]$

5.1 The effect of individual characteristics: single risk model

[...]

Thus, using Spain as the reference country, higher exit rates can be observed across the United Kingdom followed by Germany.

5.2 The effect of individual characteristics: competing risks model

[...]

Finally, regarding exits to inactivity, the United Kingdom and Germany seem to be the riskier places. 
Table 1 Departure from self-employment-Single risk models: Special focus on individual characteristics

\begin{tabular}{|c|c|c|c|c|c|c|}
\hline \multirow{3}{*}{$\begin{array}{l}\text { Number of observations } \\
\text { Number of spells }\end{array}$} & \multicolumn{6}{|c|}{$\operatorname{Prob}\left[T^{\mathrm{SE}}=j \mid T^{\mathrm{SE}}>j-1\right]$} \\
\hline & \multicolumn{6}{|l|}{26,873} \\
\hline & \multicolumn{6}{|l|}{9,753} \\
\hline Number of censored spells & \multicolumn{6}{|l|}{8,086} \\
\hline Number of completed spells & \multicolumn{6}{|l|}{1,667} \\
\hline Specification & \multicolumn{2}{|l|}{ (I) } & \multicolumn{2}{|l|}{ (II) } & \multicolumn{2}{|l|}{ (III) } \\
\hline Predicted probability (y) & \multicolumn{2}{|l|}{0.0348} & \multicolumn{2}{|l|}{0.0401} & \multicolumn{2}{|l|}{0.0379} \\
\hline Variables & $\mathrm{d} y / \mathrm{d} x$ & $t$-stat. & $\mathrm{d} y / \mathrm{d} x$ & $t$-stat. & $\mathrm{d} y / \mathrm{d} x$ & $t$-stat. \\
\hline \multicolumn{7}{|l|}{ Demographic characteristics } \\
\hline Male & -0.023 & $-6.96^{* * *}$ & -0.0199 & $-6.15^{* * *}$ & -0.0158 & $-5.13 * * *$ \\
\hline Age & -0.0069 & $-7.19 * * *$ & -0.007 & $-7.04 * * *$ & -0.0066 & $-6.88 * * *$ \\
\hline Age (squared) & $8.34 \mathrm{E}-05$ & $6.99 * * *$ & $8.65 \mathrm{E}-05$ & $6.96 * * *$ & $8.13 \mathrm{E}-05$ & $6.76^{* * *}$ \\
\hline Cohabiting & 0.0045 & $1.71 *$ & 0.0042 & 1.49 & 0.0045 & $1.69 *$ \\
\hline Number of children under 14 & $5.65 \mathrm{E}-05$ & 0.04 & 0.0003 & 0.23 & 0.0003 & 0.25 \\
\hline Relative(s) working as self-employed & -0.0079 & $-3.51 * * *$ & -0.0077 & $-3.19 * * *$ & -0.0074 & $-3.16^{* * *}$ \\
\hline \multicolumn{7}{|l|}{ Education } \\
\hline Secondary education ${ }^{(1)}$ & -0.0062 & $-2.52 * *$ & -0.0056 & $-2.11 * *$ & -0.0053 & $-2.09 * *$ \\
\hline University studies ${ }^{(1)}$ & -0.0135 & $-5.28 * * *$ & -0.0122 & $-4.32 * * *$ & -0.0124 & $-4.6^{* * *}$ \\
\hline \multicolumn{7}{|l|}{ Employment characteristics } \\
\hline Industrial sector ${ }^{(2)}$ & 0.0076 & $1.66^{*}$ & 0.0073 & 1.56 & 0.0086 & $1.84^{*}$ \\
\hline Financial services ${ }^{(2)}$ & -0.0005 & -0.13 & 0.0004 & 0.09 & 0.0007 & 0.15 \\
\hline Wholesale, hotels. restaurants \& transport $^{(2)}$ & 0.0024 & 0.72 & 0.0016 & 0.45 & 0.005 & 1.42 \\
\hline Other services $^{(2)}$ & 0.0018 & 0.4 & 0.001 & 0.21 & -0.0007 & -0.16 \\
\hline Hours of work & & & & & -0.0028 & $-6.07 * * *$ \\
\hline Hours of work (squared) & & & & & $2.01 \mathrm{E}-05$ & $5.06 * * *$ \\
\hline \multicolumn{7}{|l|}{ Previous experience } \\
\hline Previous experience as self-employed & -0.0158 & $-7.1 * * *$ & -0.0156 & $-6.17 * * *$ & -0.0146 & $-5.99 * * *$ \\
\hline Previous experience as paid-employed & -0.0055 & $-2.34 * *$ & -0.006 & $-2.42 * *$ & -0.006 & $-2.49 * *$ \\
\hline Previous experience as inactive & -0.0017 & -0.52 & -0.0021 & -0.64 & -0.0033 & -1.04 \\
\hline \multicolumn{7}{|l|}{ Previous labour market situation } \\
\hline \multicolumn{6}{|l|}{ Incomes } & $5.34 * * *$ \\
\hline Dwelling owner & & & -0.0115 & $-4.02 * * *$ & -0.0112 & $-4.05 * * *$ \\
\hline Receiving capital and property incomes (1 lag) & & & -0.0051 & $-2.1 * *$ & -0.0049 & $-2.1 * *$ \\
\hline Self-employment incomes (1 lag) (‘000) & & & -0.0008 & $-6.63 * * *$ & -0.0007 & $-6.03 * * *$ \\
\hline \multicolumn{7}{|l|}{ Duration dependence } \\
\hline Ln (Duration as self-employed) & -0.0195 & $-12.64 * * *$ & -0.0194 & $-12.02 * * *$ & -0.0182 & $-11.53 * * *$ \\
\hline \multicolumn{7}{|l|}{ Country dummies } \\
\hline Austria $^{(3)}$ & -0.0164 & $-3.37 * * *$ & -0.0193 & $-3.78 * * *$ & -0.018 & $-3.62 * * *$ \\
\hline Belgium $^{(3)}$ & -0.0134 & $-2.95 * * *$ & -0.0167 & $-3.58 * * *$ & -0.0149 & $-3.21 * * *$ \\
\hline Denmark $^{(3)}$ & -0.0047 & -0.68 & -0.0048 & -0.64 & -0.005 & -0.69 \\
\hline Finland $^{(3)}$ & 0.0019 & 0.32 & -0.0008 & -0.13 & -0.0017 & -0.31 \\
\hline France $^{(3)}$ & -0.0176 & $-4.28 * * *$ & -0.0177 & $-3.63 * * *$ & -0.0162 & $-3.38 * * *$ \\
\hline Germany $^{(3)}$ & 0.0126 & $2.1 * *$ & 0.0167 & $2.51 * *$ & 0.0195 & $2.84 * * *$ \\
\hline
\end{tabular}


Table 1 continued

\begin{tabular}{|c|c|c|c|c|c|c|}
\hline \multirow{3}{*}{$\begin{array}{l}\text { Number of observations } \\
\text { Number of spells }\end{array}$} & \multicolumn{6}{|c|}{$\operatorname{Prob}\left[T^{\mathrm{SE}}=j \mid T^{\mathrm{SE}}>j-1\right]$} \\
\hline & \multicolumn{6}{|l|}{26,873} \\
\hline & \multicolumn{6}{|l|}{9,753} \\
\hline Number of censored spells & \multicolumn{6}{|l|}{8,086} \\
\hline Number of completed spells & \multicolumn{6}{|l|}{1,667} \\
\hline Specification & \multicolumn{2}{|l|}{ (I) } & \multicolumn{2}{|l|}{ (II) } & \multicolumn{2}{|l|}{ (III) } \\
\hline Predicted probability (y) & \multicolumn{2}{|l|}{0.0348} & \multicolumn{2}{|l|}{0.0401} & \multicolumn{2}{|l|}{0.0379} \\
\hline Variables & $\mathrm{d} y / \mathrm{d} x$ & $t$-stat. & $\mathrm{d} y / \mathrm{d} x$ & $t$-stat. & $\mathrm{d} y / \mathrm{d} x$ & $t$-stat. \\
\hline Greece $^{(3)}$ & -0.002 & -0.53 & -0.0048 & -1.28 & -0.0051 & -1.42 \\
\hline Ireland $^{(3)}$ & -0.0106 & $-2.54 * *$ & -0.0089 & $-1.84 *$ & -0.009 & $-1.97 * *$ \\
\hline Italy $^{(3)}$ & -0.0128 & $-4.31 * * *$ & -0.015 & $-4.78 * * *$ & -0.0157 & $-5.28 * * *$ \\
\hline Netherlands ${ }^{(3)}$ & -0.0108 & $-1.94 *$ & -0.0118 & $-1.94 *$ & -0.0121 & $-2.14 * *$ \\
\hline Portugal $^{(3)}$ & -0.0074 & $-2.08 * *$ & -0.0119 & $-3.4 * * *$ & -0.0118 & $-3.51 * * *$ \\
\hline United Kingdom ${ }^{(3)}$ & 0.0243 & $3.55 * * *$ & 0.028 & $3.96 * * *$ & 0.0258 & $3.78 * * *$ \\
\hline \multicolumn{7}{|c|}{ Reference categories: (1) No education or primary education, (2) Construction sector, (3) Spain } \\
\hline Log likelihood & $-5,791.4$ & & $-5,764.8$ & & $-5,731.1$ & \\
\hline
\end{tabular}

Notes: *** Denotes significance at the $1 \%$ level; ** denotes significance at the $5 \%$ level; * denotes significance at the $10 \%$ level

\section{Appendix}

Variable definitions are reported below.

\section{Dependent variables}
Single risk models Dependent variable equals 1 for individuals who are full-time self-employed workers in period $t$ and exit self-employment in period $t+1$. The variable equals 0 for individuals who are full-time self-employed workers in periods $t$ and $t+1$, or the information about the labour status in $t+1$ is censored.
Competing risks models
Dependent variable equals 1 for individuals who are full-time self-employed workers in period $t$ and enter paid-employment in period $t+1$. The variable equals 2 for individuals who are full-time self- employed workers in period $t$ and enter unemployment in period $t+1$. The variable equals 3 for individuals who are full-time self-employed workers in period $t$ and enter inactivity in period $t+1$. Finally, the variable equals 0 for individuals who are full-time self-employed workers in periods $t$ and $t+1$, or the information about the labour status in $t$ is censored.


Table 4 Descriptive statistics of the departure from self-employment

\begin{tabular}{|c|c|c|c|c|}
\hline Final destination & Censored & Paid employment & Unemployment & Inactive \\
\hline Number of spells & 8,086 & 799 & 363 & 505 \\
\hline \multicolumn{5}{|l|}{ Demographic characteristics } \\
\hline Males & $73.78 \%$ & $77.60 \%$ & $69.42 \%$ & $39.01 \%$ \\
\hline Average age & 39.3 years & 35.5 years & 36.9 years & 40.7 years \\
\hline Age $21-30$ years & $20.37 \%$ & $33.17 \%$ & $34.98 \%$ & $20.39 \%$ \\
\hline Age $31-40$ years & $36.76 \%$ & $39.05 \%$ & $30.58 \%$ & $31.09 \%$ \\
\hline Age $41-50$ years & $28.04 \%$ & $19.52 \%$ & $21.49 \%$ & $25.55 \%$ \\
\hline Age $51-59$ years & $14.83 \%$ & $8.26 \%$ & $12.95 \%$ & $22.97 \%$ \\
\hline No education/Very basic education & $38.76 \%$ & $43.68 \%$ & $47.38 \%$ & $45.74 \%$ \\
\hline Primary schooling/Secondary schooling & $33.38 \%$ & $32.04 \%$ & $34.99 \%$ & $33.47 \%$ \\
\hline University studies & $27.86 \%$ & $24.28 \%$ & $17.63 \%$ & $20.79 \%$ \\
\hline Cohabiting & $76.96 \%$ & $74.09 \%$ & $66.12 \%$ & $84.75 \%$ \\
\hline Average number of children under 14 & 0.68 child. & 0.72 child. & 0.6 child. & 0.62 child \\
\hline Relative(s) working as self-employed worker(s) & $29.63 \%$ & $23.89 \%$ & $29.19 \%$ & $27.72 \%$ \\
\hline \multicolumn{5}{|l|}{ Employment characteristics } \\
\hline Construction sector & $16.05 \%$ & $19.53 \%$ & $11.57 \%$ & $7.72 \%$ \\
\hline Industrial sector & $12.65 \%$ & $15.39 \%$ & $11.57 \%$ & $13.27 \%$ \\
\hline Financial services & $16.36 \%$ & $15.15 \%$ & $9.65 \%$ & $12.48 \%$ \\
\hline Wholesale, hotels, restaurants \& transport & $40.75 \%$ & $36.79 \%$ & $55.09 \%$ & $50.09 \%$ \\
\hline Other services & $14.19 \%$ & $13.14 \%$ & $12.12 \%$ & $16.44 \%$ \\
\hline Average hours of work per week & 51.2 hours & 49.7 hours & 49.4 hours & 48.7 hours \\
\hline \multicolumn{5}{|l|}{ Experience within self-employment } \\
\hline Average duration as self-employed (in years) & 6.3 years & 4.3 years & 4.4 years & 4.7 years \\
\hline Duration: 1 year & $14.44 \%$ & $17.77 \%$ & $20.94 \%$ & $21.78 \%$ \\
\hline Duration: 2 or 3 years & $26.29 \%$ & $37.05 \%$ & $37.74 \%$ & $33.67 \%$ \\
\hline Duration: $4-6$ years & $19.85 \%$ & $23.03 \%$ & $19.84 \%$ & $19.01 \%$ \\
\hline Duration: $7-10$ years & $17.73 \%$ & $14.39 \%$ & $10.19 \%$ & $11.68 \%$ \\
\hline Duration: more than 10 years & $21.69 \%$ & $7.76 \%$ & $11.29 \%$ & $13.86 \%$ \\
\hline \multicolumn{5}{|l|}{ Previous experience } \\
\hline Previous experience as self-employed & $20.06 \%$ & $12.77 \%$ & $10.19 \%$ & $16.04 \%$ \\
\hline Previous experience as paid-employed & $28.79 \%$ & $32.17 \%$ & $19.56 \%$ & $17.03 \%$ \\
\hline Previous experience as inactive & $12.42 \%$ & $11.26 \%$ & $12.39 \%$ & $20.19 \%$ \\
\hline \multicolumn{5}{|l|}{ Previous labour market situation } \\
\hline Entering self-employment from unemployment & $23.36 \%$ & $31.66 \%$ & $50.14 \%$ & $25.54 \%$ \\
\hline \multicolumn{5}{|l|}{ Incomes } \\
\hline Dwelling owner & $75.29 \%$ & $65.58 \%$ & $67.77 \%$ & $74.46 \%$ \\
\hline Receiving capital and property incomes. & $41.02 \%$ & $34.67 \%$ & $31.96 \%$ & $35.84 \%$ \\
\hline Average annual capital and property incomes & $€ 822$ & $€ 419$ & $€ 518$ & $€ 564$ \\
\hline Average annual capital and property incomes (those who receive) & $€ 2,004$ & $€ 1,210$ & $€ 1,620$ & $€ 1,574$ \\
\hline Average annual incomes as self-employed & $€ 12,835$ & $€ 8,269$ & $€ 5,929$ & $€ 7,706$ \\
\hline \multicolumn{5}{|l|}{ Country } \\
\hline Austria & $2.39 \%$ & $1.75 \%$ & $0.83 \%$ & $1.58 \%$ \\
\hline Belgium & $3.97 \%$ & $2.49 \%$ & $3.86 \%$ & $1.19 \%$ \\
\hline Denmark & $1.83 \%$ & $2.88 \%$ & $1.65 \%$ & $0.79 \%$ \\
\hline
\end{tabular}


Table 4 continued

\begin{tabular}{|c|c|c|c|c|}
\hline Final destination & Censored & Paid employment & Unemployment & Inactive \\
\hline Finland & $4.63 \%$ & $4.51 \%$ & $6.34 \%$ & $2.97 \%$ \\
\hline France & $2.88 \%$ & $2.75 \%$ & $1.38 \%$ & $1.39 \%$ \\
\hline Germany & $8.73 \%$ & $8.64 \%$ & $7.44 \%$ & $13.27 \%$ \\
\hline Greece & $14.01 \%$ & $12.02 \%$ & $21.21 \%$ & $15.84 \%$ \\
\hline Ireland & $5.17 \%$ & $4.26 \%$ & $4.13 \%$ & $4.76 \%$ \\
\hline Italy & $16.29 \%$ & $12.64 \%$ & $20.38 \%$ & $14.86 \%$ \\
\hline Netherlands & $2.98 \%$ & $2.75 \%$ & $1.37 \%$ & $0.79 \%$ \\
\hline Portugal & $9.46 \%$ & $15.27 \%$ & $5.51 \%$ & $7.52 \%$ \\
\hline Spain & $14.67 \%$ & $18.15 \%$ & $20.39 \%$ & $15.25 \%$ \\
\hline United Kingdom & $12.99 \%$ & $11.89 \%$ & $5.51 \%$ & $19.79 \%$ \\
\hline \multicolumn{5}{|l|}{ Labour market institutions } \\
\hline Start-up incentives as $\%$ of GDP & $0.0226 \%$ & $0.0205 \%$ & $0.0196 \%$ & $0.0180 \%$ \\
\hline Employment incentives as \% of GDP & $0.1463 \%$ & $0.1145 \%$ & $0.1001 \%$ & $0.0929 \%$ \\
\hline Unemployment benefits index $(0-6)$ & 4.7049 & 4.7699 & 4.6753 & 4.6897 \\
\hline Overall EPL index (0-6) & 2.5006 & 2.6815 & 2.8690 & 2.5057 \\
\hline Statutory tax rates on dividend income $(\%)$ & $35.52 \%$ & $36.93 \%$ & $38.06 \%$ & $37.19 \%$ \\
\hline \multicolumn{5}{|l|}{ Business cycle } \\
\hline National unemployment rate $(\%)$ & $8.8 \%$ & $9.9 \%$ & $11.2 \%$ & $9.9 \%$ \\
\hline \multicolumn{5}{|l|}{ Aggregated environment } \\
\hline Services sector share $(\%)$ & $52.3 \%$ & $50.1 \%$ & $51.9 \%$ & $53.4 \%$ \\
\hline Rule of law $(0-6)$ & 4.6071 & 4.6492 & 4.5337 & 4.6564 \\
\hline
\end{tabular}

